\title{
An Approach for Formalizing the Supply Chain Operations
}

\author{
Milan Zdravković ${ }^{1}$, Hervé Panetto ${ }^{2}$, Miroslav Trajanović ${ }^{1}$, Alexis Aubry ${ }^{2}$
}

${ }^{1}$ Laboratory for Intelligent Production Systems, Faculty of Mechanical Engineering, University of Niš, Niš, Serbia

Email: \{milan.zdravkovic, traja@masfak.ni.ac.rs\}@masfak.ni.ac.rs, Address: ul. Aleksandra Medvedeva 14

18000 Niš, Serbia, Phone: +381641144797

${ }^{2}$ Research Centre for Automatic Control (CRAN - UMR 7039), Nancy-Université, CNRS, Nancy, France

Email: $\quad$ [Herve.Panetto@cran.uhp-nancy.fr, Alexis.Aubry@cran.uhp-nancy.fr\} Address: CRAN, Boulevard des Aiguillettes, B.P. 70239, 54506 Vandœuvre lès Nancy, France, Phone: +33 383684426

\begin{abstract}
Reference models play an important role in the knowledge management of the various complex collaboration domains (such as Supply Chain Networks). However, they often show a lack of semantic precision and, they are sometimes incomplete. In this paper, we present an approach to overcome semantic inconsistencies and incompleteness of the Supply Chain Operations Reference (SCOR) model and hence, improve its usefulness and expand the application domain. First, we describe a literal OWL (The Web Ontology Language) specification of SCOR concepts (and related tools), built with the intention to preserve the original approach in the classification of process reference model entities and hence, to enable effectiveness of usage in original contexts. Next, we demonstrate the system for its exploitation, in specific - tools for SCOR framework browsing and rapid supply chain process configuration. Then, we describe the SCOR-Full ontology, its relations with relevant domain ontology and show how it can be exploited for improvement of SCOR ontological framework competence. Finally, we elaborate the potential impact of the presented approach, to interoperability of systems in Supply Chain Networks.
\end{abstract}

Keywords: Enterprise Information Systems (EIS), Supply Chain, Ontology, Inter-Enterprise Interoperation, Inter-Organizational Enterprise Systems

\section{Introduction}

In order to gain real benefits from Supply Chain Management, relevant systems must span full horizontal organization of enterprises and beyond - its customers and suppliers. For dealing with the complexity of such an environment, reference models play an important role. Supply Chain Operations Reference (SCOR) [1] is a standard 
approach for analysis, design and implementation of five core processes in supply chains: plan, source, make, deliver and return. SCOR defines a framework, which aims at integrating business processes, metrics, best practices and technologies with the objective to improve collaboration between partners. In this paper, we present an approach to overcome semantic inconsistencies and incompleteness of the SCOR model, by using ontologies and thus, enabling effective knowledge management in Supply Chain Network, facilitating semantic interoperability of its participants and contributing to a further improvement of the reference model.

Semantics analysis can be useful at different levels of Supply Chain Networks. First, the semantic representation of queries and information may improve the relevance of the results and thus, improve the quality of partners' selection process. It can be used instead of or in addition to usual requests representation. Second, semantics can be used to represent participants, or groups of them, leading participants to better know each other. Such information can be useful for routing the requests to other participants in order to obtain the relevant answers within a short time and with a low traffic load. Third, this information can also be used to organize the network so as to improve efficiency. This is very important for the open settings of the supply chain networks, where the traditional approaches to business process management, which attempt to capture processes as monolithic flows, have proven to be inadequate, resulting to moving research focus from process to interaction modelling [2]. All these research directions have been followed by the researchers' community but more work is needed on the interaction between all these elements and their impact on the efficiency of the global system. The use of domain ontology is already proven as beneficial for supply chain management (SCM), in the development of self-integrating SCM systems [3], or facilitating collaboration of inter-enterprise 
design teams [4], simulation of Supply Chain Network [5], or online negotiations [6], development of approaches to semantic integration of industrial information systems [7], etc. There are also influential efforts to provide the exhaustive ontology-based semantic models for SCM [8], organized in a modular way to support the reusability and maintainability of the involved micro-theories. However, it is still very hard to integrate all these efforts in a single formal theory which would enable a multiplication of achieved impacts. Ontologies are formal models of collective knowledge. The consensus on their structure is extremely hard to reach [9], particularly for a very expressive (or richly axiomatized) ontology with large number of concepts. In response to this problem, the approach of collaborative conceptualization is proposed and applied on the case of electronic product catalogues integration [10].

The development of reference models in different domains is a community response to interoperability problems. They aim at the standardization of domain collaboration by providing categorization schemes or taxonomies - knowledge structures, interpreted in organized way - to be used as guidelines in the collaboration of humans and systems.

Like most of the other reference models, SCOR is a form of knowledge organization system. The key feature of these models is subjectivity, or contextdependent determination [11]. They are not developed with the intent to be semantically rich or precise, but to provide human-understandable knowledge on the specific domain. However, their implicitness is considered as an obstacle for a machine-based interpretation. SCOR lacks semantic precision. SCOR's Input/Output entity entails all resources exchanged between process elements and actors - physical or non-physical, states, events, documents, etc. System entity includes information 
systems, modules, capabilities, approaches or volume of use, integration levels, etc. Sometimes, reference models do not provide enough expressivity for a complete formal model. In the case of SCOR, this is evident from the lack of relationships between metrics and systems, which could point out to the source of information needed for performance measurement.

Industrial reference models are not formal models. They are descriptive languages. They were created with an objective to aggregate entities for some purpose, rather than to describe the nature of the entities [12]. Hence, they are very hard to maintain and to evolve in a consistent way. Dynamics and volatility of concepts are much easier to manage if they are represented by a set of meaningful statements or expressions, rather than by narrative descriptions. Also, higher levels of expressivity and axiomatization extend the opportunities for automated support. However, industry acceptance of the reference models shows that practical benefits are more likely to be achieved when they are focused on highly contextualized approaches where formalizing domain knowledge is involved. Domain knowledge evolves at highest rate at lower levels of abstraction, in domain community interaction, where consensus is more likely to be reached.

Enterprise formal models usually stand at the opposite end of the abstraction ladder. Although there are many enterprise modelling frameworks used in an industry, this is not the case with enterprise ontologies. In this paper, we present three existing enterprise ontologies with different levels of expressivity and show how one of these can be used for improving the competence of the semantically enriched SCOR model. Although these ontologies had significant impact to enterprise modelling scientific community and some briefly reported applications, there are no strong evidences of their industry acceptance with their intended purpose. While obvious lack of practical 
implications can be justified by the technology-related implementation difficulties, it is a fact that many of the existing efforts in development of the common enterprise model are based on an inspirational approach to enterprise modelling, implemented by the groups of experts, not by community [12]. Moreover, enterprise ontologies are usually created from scratch. As a result, the development and, especially validation processes take a very long time to complete [13], due to a typically large amount of work needed for analysis and synthesis of the domain knowledge, as well as achievement of the consensus on developed conceptualizations within the relevant community. However, they do provide a formal basis for semantic interoperability of systems. Thus, we consider the coherence between creation, evolution and use of specific, highly contextualized knowledge and development of formal expressive models as a very important factor for the usefulness of domain ontology.

In this paper, we present the approach to semantic enrichment of the SCOR reference model, for the benefit of enhanced interoperability of resulting ontologies, on multiple layers of expressivity and abstraction. This approach extends already verified model and maps its original elements to their semantics. It secures the integrity of existing standard by using multiple levels of models' expressivity. Section 2.1 and 2.2 of this paper describe the ontology development issues, relevant for systems interoperability, and some development decisions made in the conceptualization of the enterprise modelling domain and SCOR model formalization. In Section 2.3 we show how our approach layers the implicit knowledge, application and domain representation models. This approach is demonstrated by developing semantically aligned models (or model layers) of: a) implicit knowledge on the Supply Chain operations, namely, SCOR reference (SCOR-KOS - SCOR Knowledge Organization System); b) specific application (problem domain) ontology (SCOR-Cfg 
for process configuration) and c) micro theory for supply chain operations (SCORFull), which semantically enriches the SCOR reference model. These models are described in detail in Section 3. The main intention behind the decisions made in the implementation of this formalization approach is to facilitate the semantic interoperability of systems, relevant for Supply Chain Networks management. Hence, in Section 4, we elaborate on the notion of semantic interoperability of systems and describe the potential impact to the architecture of the relevant enterprise information systems and some expected practical benefits.

\section{Theoretical Background}

\subsection{Approaches to ontology development and interoperability}

One of the main issues of efficient use of information systems is interoperability between multiple representations of reality stored within the systems and/or between these representations and systems' users, namely, their perceptions of reality [14]. This issue is expected to be resolved by use of ontologies - logical theories for formal, explicit, partial specification of conceptualization [15].

Interoperability of information systems depends on the quality and mutual consistency of the underlying ontologies [16]. Differences in conceptualizations (or paradigmatic stands) to which ontologies are committed can cause semantic mismatch, and hence, have a negative effect on interoperability. Namely, in ontology development, knowledge workers or domain experts can choose descriptive or prescriptive approach, temporal or static representations, objective or subjective stand, etc. Negative effects of the inconsistent conceptualizations can be reduced by employing additional efforts in mapping, alignment, translation, transformation or merging the corresponding ontologies [17]. 
Using different levels of granularity is a common approach to engineering of an ontological framework. It is applied in building upper ontologies, combining continuant, enduring perspectives of reality and concepts extended through time [18]. Four-dimensional perspective on reality within a single framework can be granularized further to strategic, operational and tactical sub-perspectives. A variety of granularity levels in an ontological framework extends the scope of inference. Also, it contributes to achievement of the semantic interoperability between corresponding systems.

\subsection{Enterprise and supply chain ontology}

TOVE ontology [19] applied a formal approach to enterprise modelling, representing activities, states and time (top-level ontology), organization, resources, products, costs and activity-based cost management. The primary focus of the TOVE enterprise model has been in linking the structure and behaviour through the concept of empowerment - the right of an organization agent to perform status changing actions. TOVE aims at providing sophisticated support to decision making by enabling the inference, not only on basis of what is explicitly stated in the model, but also on the basis of what is implied by the model. It introduces the notion of an ontology competence and corresponding competency questions - the ontology benchmarks, in the sense that the ontology is necessary and sufficient to represent the tasks specified by the competency questions and their solution. Thus, TOVE organizational ontology

defines three sets of competency questions: 1) Structure; 2) Behaviour; and 3) Authority, empowerment and commitment competency.

The Enterprise Ontology [20] is a result of the work in development of a method and a computer tool set for enterprise modelling, based on a formal models. It aims at ensuring that all parties, involved in the enterprise have a shared understanding of the 
relevant aspects. Its role is to act as a communication medium (primarily, but not exclusively - between humans). Secondly, it is intended to assist acquisition, representation and manipulation of enterprise knowledge. Thirdly, it is intended to enable the interoperability, by using the ontology as an interchange format for terms related to business enterprise. The main criteria for selection of the terms were common usage (consensus on the meaning) and avoid of ambiguity. The building blocks on the Enterprise Ontology are notions of an Entity, a Relationship, a State of Affairs and a Role. These are the primitives which are used for expressing the definitions in an ontology and they are referred to as concepts of "meta-ontology". They are specialized to the concepts of 4 sections: 1) Activity, Plan, Capability and Resource; 2) Organization; 3) Strategy; and 4) Marketing.

IDEON $^{\mathrm{TM}}$ ontology [21] is one of the candidate ontologies for modelling collaborative distributed enterprises. It employs four complementary perspectives to capture the key concepts and relationships of the enterprise. First, the enterprise context view represents the interaction between an enterprise and its external environment (partners, customers, competitors, etc.). It introduced the interesting concept of "sensors", employed for observing the environment, enabling the enterprise to act upon the assessment of the observation, with a goal to achieve a specific effect to its environment. Second, the enterprise organizational view captures its inner structure, assigns goals, and selects strategies for their achievement and acts upon them, by employing relevant processes. Third, the process view represents planning-execution-control cycle. Fourth, the resource view elaborates on the various types of resources that might be needed to execute a process.

Table 1 show the comparative overview of the above enterprise conceptualizations, which illustrates used development approaches (purpose, 
modularization decisions) and implementation features (expressivity, notations, applications).

\begin{tabular}{|l|l|l|l|}
\hline & $\begin{array}{l}\text { The Enterprise } \\
\text { Ontology }\end{array}$ & TOVE & IDEON \\
\hline $\begin{array}{l}\text { Purpose } \\
\text { (motivation) }\end{array}$ & $\begin{array}{l}\text { Enterprise } \\
\text { modelling }\end{array}$ & $\begin{array}{l}\text { Enterprise } \\
\text { modelling }\end{array}$ & $\begin{array}{l}\text { Collaborative } \\
\text { enterprise } \\
\text { modelling }\end{array}$ \\
\hline $\begin{array}{l}\text { Domains } \\
\text { (modularization } \\
\text { approach) }\end{array}$ & $\begin{array}{l}\text { 1) Activity, plan, } \\
\text { resource, 2) } \\
\text { Organization, 3) } \\
\text { Strategy, 4) } \\
\text { Marketing }\end{array}$ & $\begin{array}{l}\text { 1) Structure, 2) } \\
\text { Behaviour, 3) } \\
\text { Authority, } \\
\text { empowerment and } \\
\text { commitment }\end{array}$ & $\begin{array}{l}\text { 1) Interaction, 2) } \\
\text { Organization, 3) } \\
\text { Process, 4) } \\
\text { Resource }\end{array}$ \\
\hline Expressiveness & Low & High & $\begin{array}{l}\text { OWL } \\
\text { (Web Ontology } \\
\text { notations }\end{array}$ \\
$\begin{array}{l}\text { Ontolingua } \\
\text { Language) }\end{array}$ & $\begin{array}{l}\text { Unified Modeling } \\
\text { language (UML), } \\
\text { Knowledge } \\
\text { Interchange Format } \\
\text { (KIF) }\end{array}$ \\
\hline Known applications & $\begin{array}{l}\text { The Enterprise } \\
\text { Toolset (Business } \\
\text { Process models, } \\
\text { Agent-based } \\
\text { architecture for } \\
\text { integration) }\end{array}$ & $\begin{array}{l}\text { Integrated Supply } \\
\text { Chain } \\
\text { Management, } \\
\text { Process integration } \\
\text { in Enterprise } \\
\text { Engineering }\end{array}$ & $\begin{array}{l}\text { Process-centric } \\
\text { crisis action } \\
\text { planning and } \\
\text { execution, } \\
\text { Integrated Product- } \\
\text { Process } \\
\text { Development } \\
\text { (IPPD) }\end{array}$ \\
\hline
\end{tabular}

Table 1. Overview of the enterprise ontologies

To the best of our knowledge, there were only a few attempts to ontologize SCOR model. SCOR+ [22] is directed towards overcoming the limitations of the basic SCOR model through an ontology based tool. This tool will enable an automated and comprehensive definition of the supply chain at four of its distinctive levels: supply chain level, the enterprise level, the elements level, and the interaction level. It enables generation of generic explicit views and models that represents the four levels. Unfortunately, SCOR+ is a proprietary product and details on the formalization approach are not accessible. Lin [23] extended the SCOR model by generalizing existing elements to 3A concepts (Activity, Artefact, Actor-Role), defined in GPO (General Process Ontology). Also, she used the model for 
development of the goal ontology, by modelling SCOR performance attributes as general soft goals and deriving domain specific goals from attributes' metrics. Vegetti et al [24] used SCOR to develop the SCOntology. They extended SCOR with the notions of an enterprise model, with aim to provide the foundations for the specification of information logistics processes in extended supply chains associated to process industries. Considering that enterprises are collaborating for a certain purpose, supply chain may be considered as a kind of system-of-networked enterprises. Lu et al [25] extended the ONTO-PDM Product Ontology developed by Tursi et al [26] with the SCOR model. The resulting ONTO-SCOR ontology is then defining a product-centric supply chain ontology for facilitating the interoperation between all enterprise applications involved in an extended supply chain. On basis of the analysis of the contribution of the SCOR model to the alignment of business processes and information systems, Millet et al [27] proposed the extended reference model, including the structure of information exchanged between processes. This model is proposed in response to the identified weaknesses of the current SCOR model, in specific, lack of important process dependencies.

In addition, there are many relevant papers with reported work on other reference models' formalization, addressing the semantics of RosettaNet [28], UNSPSC [29, 30], AIAG and STAR [31], EDI [32], etc. Presented results, methods, tools and gained experiences were extremely useful in setting up and implementing this approach.

\subsection{Description of our approach}

Our approach builds upon three of the five general approaches to ontology design: inspiration, induction, deduction, synthesis and collaboration [33]. Induction is used in the phase of semantic enrichment of a reference model, by improving the semantic 
precision of the categorizations. It is combined with inspirational approach which is characterized by an individual (authors') viewpoint about the used abstractions. Inspiration is also used for formalizing problem solving models - application models, based on the design goals. Finally, synthesis is employed in mapping of a semantically enriched model with relevant ontologies, with the aim to enable semantic interoperability and/or to extend an inference scope.

In order to formalize the reference model, we propose the approach of using the semantically aligned layers of a literal specification of a reference model, its semantic enrichments and resulting domain ontology and application models, developed on basis of the different design goals. Approach reflects the practices from AI domain of using the different granularities of domain knowledge in solving engineering problems of different abstraction [34]. The approach is demonstrated, in the next section, on the example of SCOR model and its application for generating process thread diagrams.

Figure 1 shows the framework for semantic enrichment of reference models, and include source data (reference models and existing domain ontologies), various tools, and resulting models: literal OWL specification, semantically enriched model and application ontologies.

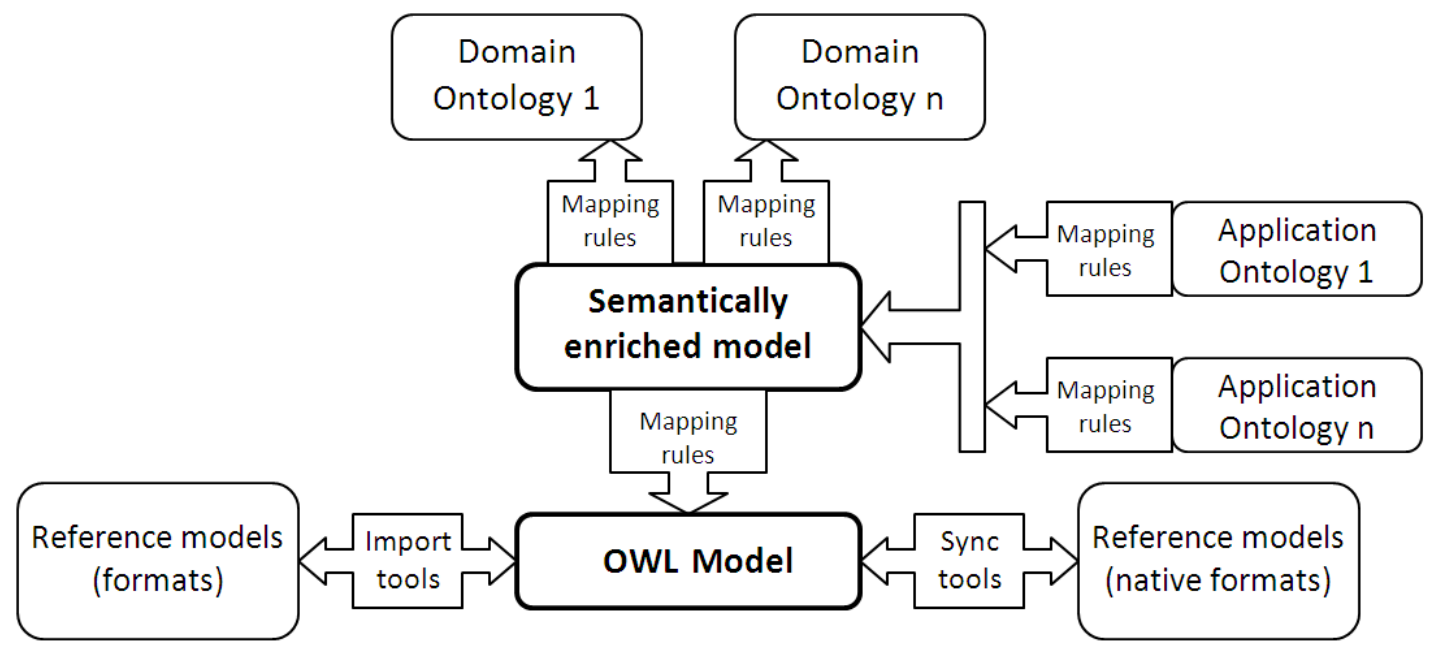


Figure 1. Framework for semantic enrichment of reference models

As reference models are stored in number of different formats and representations, the use of import facilities in support to initial development and continuous update of the OWL model is recommended. Some of the examples of the import tools are EulerGUI ${ }^{1}$, a lightweight IDE that translates UML XMI format and XML schemas into N3 triples and Anzo for $E x e^{2}$, which extracts RDF data from Excel spreadsheets. Update of the models or instantiation of relying concepts can be automated when the import tools and respective API's are used for alignment of OWL models and native data formats of the applications which are using the reference models. In case of SCOR, some of the examples of such applications are ARIS EasySCOR by $\mathrm{IDS}^{3}$ or e-SCOR by $\mathrm{Gensym}^{4}$, used for the benefit of SCOR implementation process.

Layering of application and domain representation models reflects the paradigm of separation of domain and task-solving knowledge [35] and assume their mutual independence [36]. Thus, arbitrary design goals can be defined, formalized to set of competency questions and used for development of a task-solving, application ontology. Although problem domain is restricted to a supply chain context, level of its variety can be extended in process of synthesis, namely, mapping of semantically enriched model with other relevant ontologies, such as enterprise ontology, TOVE ontologies, and others.

\section{Semantic enrichment of SCOR model}

\footnotetext{
${ }^{1} \mathrm{http} / / /$ eulergui.sourceforge.net

${ }^{2} \mathrm{http} / / / \mathrm{www} . c a m b r i d g e s e m a n t i c s . c o m /$ semanticexchange

${ }^{3}$ http://www.idsscheer.com/ru/ARIS/ARIS_Reference_Models/ARIS_EasySCOR/115741.html ${ }^{4} \mathrm{http}: / / \mathrm{www}$. gensym.com
} 
For building the fully expressive SCM semantics, we start from the obvious point of community consensus - reference models, in specific - SCOR. Because of the SCOR's weak semantics, in the first step, we model it as a knowledge organization system (KOS), but we use semantic tools to represent this model in a computerizable language - OWL (Web Ontology Language) language. OWL [37] is a family of knowledge representation languages, endorsed by the World Wide Web Consortium, which provides the syntax for authoring and exchanging the ontologies among relevant tools and applications. Figure 2 shows entities of SCOR-KOS OWL model and relationships between them.

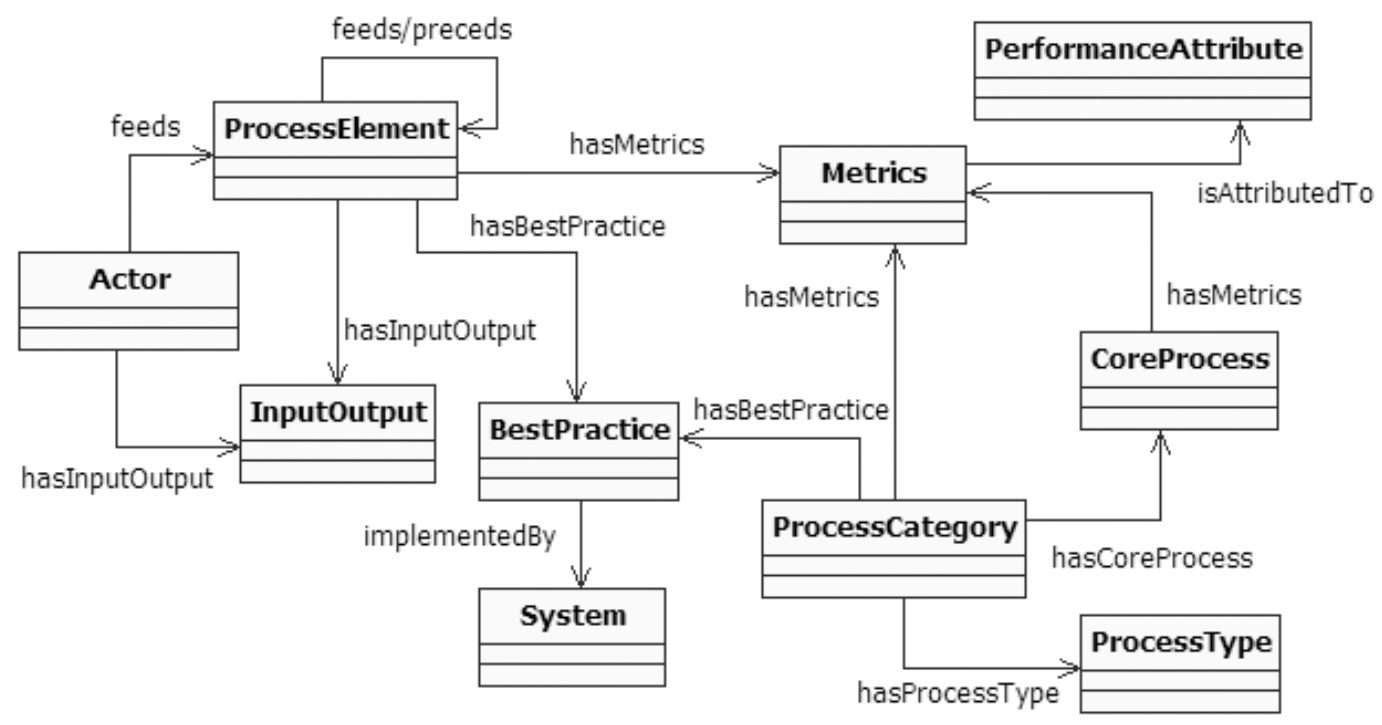

Figure 2. Entities of SCOR-KOS OWL model

Aim of the literal OWL specification is to preserve the classification approach and represents model's concepts and properties and thus enables the use of a resulting SCOR-KOS model for the original purpose. This purpose can be formalized by the competency questions, used for the validation of resulting model. Competency of a SCOR-KOS OWL model is validated by using following questions: which process elements constitute one SCOR process and in which order? What are the input and output resources for the selected process element? What are the metrics and best 
practices for the selected process element? Which systems can facilitate the improvement of the selected process element and/or process category?

The actual order of process elements is determined by executing SPARQL queries against asserted "precedes" (meaning direct precedence) triples. The definition of concurrency in a SCOR-KOS OWL model is used only for the determination of flows branching and hence, it is not semantically correct. Concurrency is inferred on basis of "isConcurrentWith" relation and modelled by property chain axioms, on basis of asserted "precedes" and inferred (inverse) "succeeds" property:

precedes o succeeds $=>$ isConcurrentWith

, or by using RDQL query:

$\exists$ precedes. $(\geq 2$ succeeds $)$

Flows of input and output resources are determined by SPARQL queries, which return instances of "SCOR_InputOutput" concept from domain of asserted triples of "hasInput" and "hasOutput" properties. The source of these properties is determined from the domain of "fedBy" property, which is used to assert connections between process elements from different process categories. Inference of systems which can facilitate improvement of selected process elements (categories) is achieved by implementing properties:

implements(SCOR_System,SCOR_BestPractice), and:

facilitates (SCOR_BestPractice,SCOR_ProcessElement),

as inverse to "implementedBy" and "hasBestPractice", used for the assertion of relationships between process elements, best practices and systems. The properties above are defined as sub-properties of transitive property "enable", hence, enabling reasoning of relationships between "SCOR_System" and "SCOR_ProcessElement". 
By defining inverse property "enabledBy", the inference on relationships between systems and process elements (categories) becomes possible in the opposite direction. Thus, it is possible to identify systems which can improve the performance of a selected process element and/or category.

SCOR-KOS OWL is used for the development of the web application for browsing and visualization of the SCOR framework. Main features of the web application are: display of the selected process category map, display of the input/output resources (including sources/destinations), best practices and metrics for selected process element and customization of the display. Figure 3 shows the web application's work area, with displayed output resources, best practices and metrics for „P4.04. Establish delivery plans“ process element of „P4. Plan Deliver“ process category.

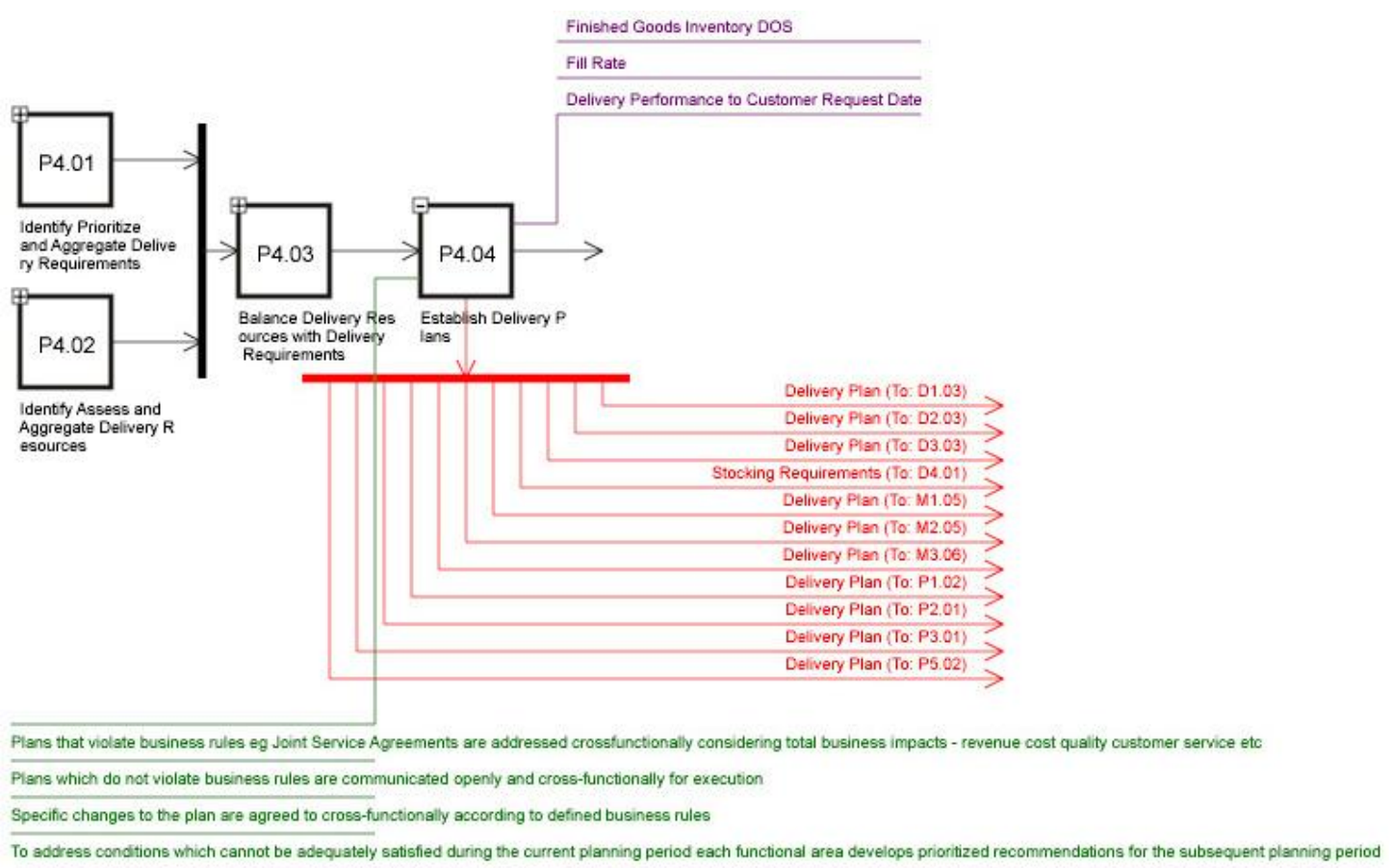

Figure 3. „P4. Plan Deliver“ process category

\subsection{SCOR-Cfg Application Ontology}


For demonstrating the approach, a design goal has been set - generation and representation of a SCOR thread diagram. A thread diagram is a standard tool used in implementation of a SCOR model. In this case, it is inferred as configuration of source, make and deliver processes, on basis of asserted product topology, participants and production strategies for each component. Different process patterns (and roles) are inferred as a result of SPARQL queries executed against SCOR-Cfg model in each of the three possible manufacturing strategies: made-to-stock, made-toorder or engineered-to-order.

The approach is demonstrated on a simplified case of snow making facility product engineering, which involves purchase of fan guns (from stock), hydraulic equipment (by order) and sourcing engineering and production of a pump house. Figure 4 shows the basic interface for the definition of snow making facility product topology and generated SCOR thread diagram.

\section{Supply Chain Definition}

Selected project: Snow-making-facility-engineering

CHANGE OR CREATE NEW

Principal product topology

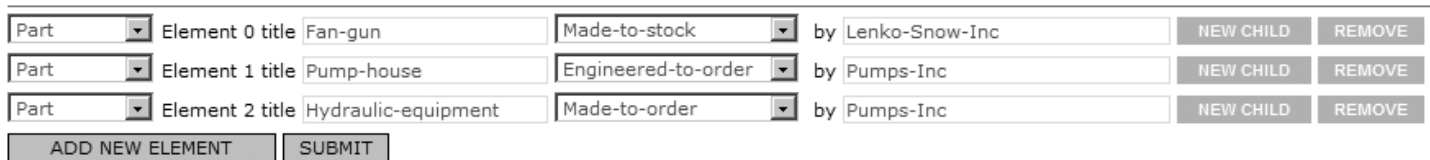

SCOR Thread Diagram

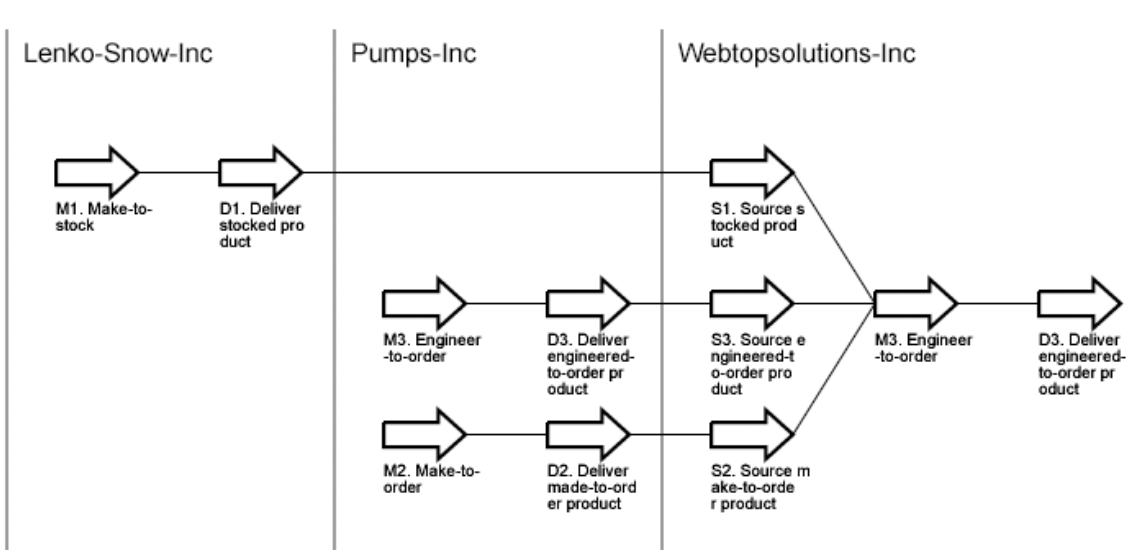


Figure 4. Web interface for definition of a product topology and generated SCOR thread diagram

Product configuration is asserted to application ontology: SCOR-Cfg OWL model, consisting of following concepts: SC_project, SC_product, SC_production_type, SC_process (with child concepts, corresponding to different process types) and SC_participant. The generation of a SCOR thread diagram is done by selecting (and rendering) participants of supply chain project, its products (components) and, finally, processes, in exact order.

Main features of the web application are: development of complex thread diagrams, generation of process models and workflows and generation of implementation roadmap.

First, the above example shows only interactions and collaborations between customer and its first-tier suppliers. The number of visualized levels depends on the submitted product topology: if the detailed product topology is entered, the full supply chain would be represented, with the number of tiers corresponding to the depth of a product topology. Also, the horizontal organization of individual supply chain actors can be represented in more detail, by inferring additional participants for different manufacturing strategies: warehouses $(\mathrm{D}, \mathrm{S})$, plants $(\mathrm{M})$ and headquarters $(\mathrm{P})$.

Second, a SCOR thread diagram is not a process map. In fact, it is just a representation of a supply chain configuration. The full process model can be generated by adding new rules for configuration of the SCOR PLAN activities and by exploiting alignment relations between the SCOR-KOS and SCOR-Cfg OWL models.

Third, alignment relations between the SCOR-KOS and SCOR-Cfg OWL models also provide opportunities for the generation of a detailed implementation roadmap, consisting of proposed best practices, relevant systems (or their modules, capabilities, intended use, etc.) for their execution, resource tracking (SCOR Inputs 
and Outputs) and environment for measuring the performance of a supply chain, by using the SCOR metrics.

\subsection{SCOR-Full Ontology}

SCOR-Full is a domain ontology - a micro theory for representation and management of knowledge of the supply chain operations. It formalizes core concepts of supply chain operations, embedded in SCOR model definitions, and is developed by semantic analysis of SCOR Input/Output elements, identification of core terms and their categorization. It extends SCOR-SYSTEM ontology, which formalizes the SCOR System element. It is extended by SCOR-GOAL ontology, which semantically maps its concepts to SCOR Performance Metrics element. These ontologies are not in the scope of this paper and will not be elaborated.

SCOR-Full ontology does not aim at formalizing the supply chain, but only to resolve semantic inconsistencies of a SCOR reference model. Thus, its scope is strictly limited to using the common enterprise notions for expressing the existing elements of SCOR model. Central notion of the SCOR-Full ontology (as it is the case for SCOR model) is a generalization of process, in the sense that it acts as the main context for semantic definition of other concepts in the ontology.

Main concepts of the SCOR-Full ontology are: Agent, Course, Resource Item (and its sub-concepts: Information Item, Physical Item, Configured Item and Communicable Item), Function, Quality and Setting. Figure 5 shows the main concepts of SCOR-Full ontology and relationships between them. 


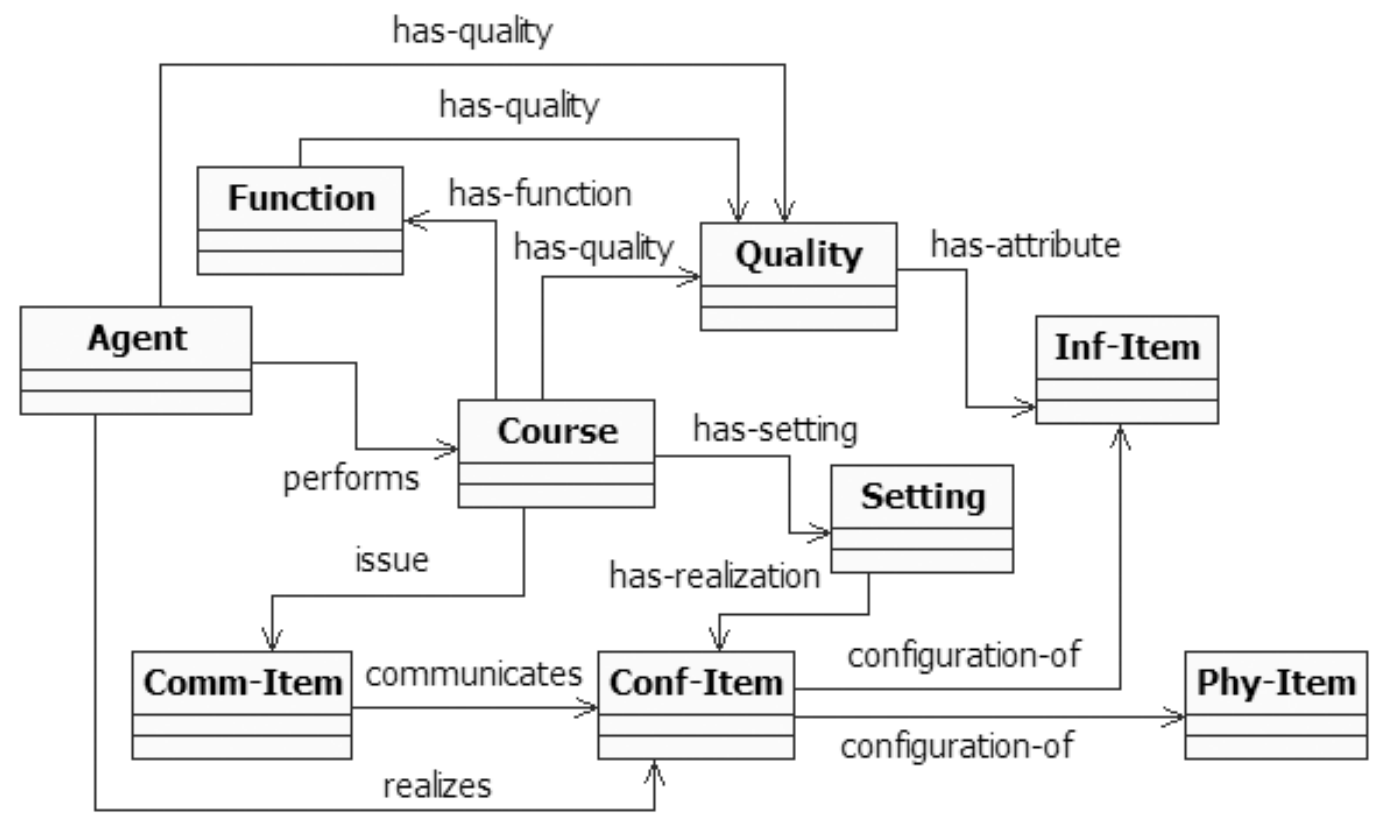

Figure 5. Main concepts of SCOR-Full ontology and relationships between them

Agent is the concept which describes an executive role and entails all entities which perform individual or set of tasks within the supply network, classified with the concepts of equipment, organization, supply chain, supply chain network, facility and information system. Although semantically described as roles, agents do not have explicit definition of functions. Functionality is defined as a property of a course, performed by an agent. Hence, agents are functional in a context of a course they execute.

Course classifies prescriptions of ordered sets of tasks: activity, process, method, procedure, strategy or plan, at the same level of abstraction. The notion of course generalizes "doable" things with common properties of environment (enabling and resulting states, constraints, requirements, etc.), quality (cost, duration, capacity, performance, etc.) and organization (agent and business function).

Setting concept provides the description of environment of a course. It aggregates semantically defined features of the context in which course take place - 
its motivation, drivers and constraints. Thus, it classifies rules, metrics, requirements, constraints, objectives, goals or assumptions of a prescribed set of actions.

Quality is the general attribute of a course, agent or function which can be perceived or measured, eg. capability, capacity, availability, performance, cost or time/location data.

Function concept entails elements of the horizontal business organization, such as stocking, shipping, control, sales, replenishment, return, delivery, disposition, maintenance, production, etc.

Instead of representing process flows, SCOR-Full is used to model enabling and caused states of the relevant activities. These states are represented by the concept of configured item (Conf-Item), the range of the has-postcondition and hasprecondition properties of Course and its sub-concept - Activity.

A resource item is a general term which encloses communicated (Comm-Item, e.g. Notification, Response, Request) and configured (Conf-Item, with defined state) information items (Inf-Item), such as Order, Forecast, Report, Budget, etc., and physical items (Phy-Item). Where information items are the attributes of a Quality (of Function, Agent or a Course), their configurations are realizations of the rules, metrics, requirements, constraints, goals or assumptions of a course.

Configured items model state semantics of the resource - physical or information item, the notions which are used to aggregate the atomic, exchangeable objects in enterprise environment. Examples of information items are Order, Forecast, Budget, Contract, Report, Proposal, Bill-Of-Material, etc. Their structure is not addressed by SCOR-Full ontology - from this perspective, these are the atomic concepts which can be semantically defined when mapped to other enterprise ontologies. Physical items are Product (MRO-Product, Defective-Product and Part) 
and Scrap. Configured items are characterized by one or multiple states of information or a physical item:

$$
(\text { Phy-Item(?x) } \vee \text { Inf-Item(?x)) } \wedge \text { has-state(?x,state(?y)) } \Rightarrow \text { Conf-Item(?x) }
$$

Available states are identified in the analysis of SCOR model and include 25 possible attributes of the configured item, which can be associated to different information and physical items. Some of the examples of the states are: Adjusted, Approved, Authorized, Completed, Delivered, Installed, Loaded, Planned, Released, Returned, Updated, Validated, etc.

Where Inf-Item defines the semantics of the relevant resource, Conf-Item describes its dynamics. Note that SCOR-Full asserts the semantic relation (realizes (Agent, Conf-Item)) which can be used to infer which Agent is responsible for a particular state of the resource, although this specific information cannot be extracted from the original SCOR model. SCOR-Full will rely on the external enterprise knowledge to fill this and other gaps.

For the expressive process model, it is crucial to define how resources are communicated among activities and their corresponding actors. This knowledge is embedded (explicitly or implicitly) in original SCOR model (in natural language) and is used by SCOR-Full ontology to formalize abstract communicated item (CommItem) which aggregates specific concepts of Notice (or its child concept - Signal), Request, Response and Receipt. SCOR model does not provide explicit information about who communicates configured items but this can be inferred by using external knowledge when property chain of performs(Agent, Course)o issue(Course, Comm-Item) is exploited, where former relation is inferred on basis of the mappings with external ontologies and latter - from SCOR-KOS OWL. Necessary conditions for a 
Comm-Item are that it is issued (requested, responded, notified or received) by a course and that it communicates a configured item:

$$
\text { Course }(? x) \wedge \text { Conf-Item }(? y) \wedge \text { issue }(? x, ? z) \wedge \text { communicates }(? z, ? y) \Rightarrow \text { Comm- }
$$
$\operatorname{Item}(? \mathrm{z})$

More specific axioms are set for the sub-concepts of Comm-Item, by using the sub-properties of issue (Course,Comm-Item) property, namely: issue-request(Course, Request), issue-response(Course, Response), issue-notice(Course, Notice) and issuereceipt(Course, Receipt).

Currently, SCOR-Full ontology has 212 concepts and 32 properties and is semantically mapped to the SCOR Input/Output elements. In order to increase the flexibility of semantics framework, SWRL (Semantic Web Rule Language) rules are used for mapping the SCOR-Full concepts to SCOR-KOS OWL instances. For example, all instances of the business-rule class from SCOR-Full ontology are the same as SCOR Input/Output concept "Business_Rules_For_Return_Processes", if there exists a return process in SCOR-Full ontology which has a business rule from above, as a setting:

$$
\text { business-rule(?x) } \wedge \text { return-process(?y) } \wedge \text { has-rule(?y, ?x) } \Rightarrow \operatorname{SameAs(?x,}
$$

Business_Rules_For_Return_Processes)

Semantic mappings between SCOR-Full and SCOR-KOS enable characterization of supply chain operations managed by using SCOR-Full ontology, in context of SCOR reference model. For example, based on the above SWRL implication, it can be inferred that a business rule, which is asserted in SCOR-Full ontology as a setting for an instance of the return process, is an output of the SCOR process element ER.01 Manage Business Rules for Return Processes. In the opposite 
direction, relevant inferences of SCOR-KOS OWL model can result with a formal semantics of the selected SCOR element.

SCOR-Full ontology is expected to support knowledge management in supply chain operations. It classifies concepts and relevant data objects, to be used in collaborative systems, such as Semantic information pool for manufacturing supply networks (SIP4SUP) [38], currently in development. It enables lookup of data objects, required for consistent and complete definition of supply chain operations concepts. It provides a roadmap for implementation of SCOR reference model. It does not improve the expressivity of SCOR, because it only uses common enterprise notions and proposed generalizations to formalize core concepts of supply chain operations, embedded in SCOR model definitions. However, these generalizations enable alignment of SCOR-Full model with relevant enterprise models, such as TOVE ontology and thus, exploitation of its knowledge for improving the competence of SCOR. Last, and most important, SCOR-Full ontology is expected to facilitate the semantic interoperability of systems, relevant for Supply Chain Networks management.

Where SCOR-KOS provides implicit semantics of the supply chain operations by using a semantics representation language, SCOR-Full (and its corresponding mappings with the domain ontologies and SCOR-KOS itself) makes this semantics explicit. Objective conceptualization and corresponding explicit representation of domain knowledge is considered as a main condition for making the relevant systems semantically interoperable. In the next section, we discuss on the semantic interoperability in Supply Chain Networks. We show how the presented approach can be exploited to improve the expressivity of the underlying ontological framework and 
describe some practical impact of the semantically interoperable systems to Supply Chain Management.

\section{Ontologies and semantics issues in Supply Chain Networks interoperability}

Supply Chain Networks may be considered, to a certain extent, as systems-ofnetworked systems. The System-of-Systems (SoS) paradigm is widely recognized and has become quite studied since a decade [12], as it has potentially practical applicability in systems engineering. SoS-organized systems, such as Supply Chain Networks, could make efficient use of resources from a variety of domains. One of the main characteristics of SoS is what authors are calling the "connectivity" property [39]. "Connectivity" refers to interoperability of the many suppliers taking part in Supply Chain Network.

Interoperability is typically defined as the ability of two or more systems or components to exchange and use information [40]. Integration is generally considered to go beyond mere interoperability to involve some degree of functional dependence [41]. Integration is desirable within the horizontal organization of the single enterprise or, in some cases, between focal partner and first-tier (strategic) suppliers (for example, with third-party logistics partners). However, in lower levels of supply chain, the tight couplings can produce unacceptable outcomes, mostly related to decrease of flexibility. The main prerequisite for achievement of interoperability of the loosely coupled systems is to maximize the amount of semantics which can be utilized and make it increasingly explicit [42], and consequently, to make the systems semantically interoperable.

While database interoperability has been widely studied by the research community, it takes into account technological concerns. Interoperability in supply chain is mainly related to semantics issues, where the semantics is discussed in 
multiple levels of abstraction, as well as in multiple contexts. It needs to align strategic views, expressed in enterprise architecture, with business process organization and control, and consequently, with IT architecture and infrastructure [43]. Many researches are trying to demonstrate that semantic interoperability can be enabled through setting up an Ontology. The use of ontology is required as it acts as a conceptual model representing enterprise consensus semantics [44]. It aims at reducing the semantics loss among heterogeneous information systems that are sharing mostly common concepts from the same area of knowledge. Furthermore, ontology provides a common understanding and a formal model of the basic entities, properties and relationships for a given domain that are essential to overcome semantic heterogeneity. Semantic interoperability ensures that the meaning of the information that is exchanged is automatically interpreted by the receiver of a message. In centralized systems, this property improves the relevance of query answers. In distributed heterogeneous systems, it is compulsory to enable autonomous heterogeneous sources understand each other to obtain relevant results [45].

Many works rely on the assumption that a single ontology is shared by all the participants of the system. However, in systems-of-systems with autonomous subsystems, this assumption is not realistic anymore. On the contrary, one has to consider that the participants create their ontologies independently of each other. Thus, most often the ontologies differ. Still, the distinctness of ontologies does not prejudice logical inconsistency of their terms, especially if they focus on different contexts of the same concepts. Namely, ontology is not a tool for checking correctness of reality, but for its subjective or objective representation. To tackle this problem, research on ontology matching proposes several techniques to define correspondences between entities of two ontologies. So, in some way, ontology matching highlights the shared 
parts of two ontologies. Thus it provides the basis for interoperability between heterogeneous systems and by "transitivity" in the whole system [46]. Typically, correspondences between two interacting ontologies are expressed by logical equivalences, subsumption or sameness relations, assertions of constraints, based on the object properties or identification of rules, with the form of logical implication between the antecedent and consequent statements.

Also, meanings from ontologies, developed in isolation, can be reconstructed or re-created by using contextualization or logical theories, such as ontology of descriptions and situations (DnS) [47], which enable the first-order manipulation of micro-theories and models, independently from an upper ontology.

\subsection{Interoperability and expressivity of the formal model}

SCOR shows lack of expressivity for a complete formal model. One of the evidences is the lack of relationship between metrics and systems, which could point out to the source of information needed for performance measurement. This is obvious limitation of the reference model and it can not be addressed in the process of semantic enrichment, as this relationship does not exist.

However, semantically enriched model enables us to establish the references between formalized systems, system capabilities, intended uses, etc., and goals, mapped to the metrics of the SCOR model, by using the external knowledge, formalized in various domain ontologies. Namely, if there exist systems S1 and S2, driven by the ontologies $\mathrm{O}_{1}$ and $\mathrm{O}_{2}$ (external knowledge), and if there exist alignment between these ontologies $\mathrm{O}_{1}=\mathrm{O}_{2}$, the competence of $\mathrm{O}_{1}$ will be improved and $\mathrm{S}_{1}$ will be enabled to make more qualified conclusions about its domain of interest.

For example, in TOVE organization ontology, the concept of CommunicationLink (cl) captures the notion of benevolent communication in which organization 
agents voluntarily provide information that they believe are relevant to other agents. TOVE organization ontology can be extended with a property chain axiom of the new information-provided-by(inf,oa) relationship, established between the concepts of Organization-Agent (oa) and Information (i):

information-provided-by(inf,oa) $\equiv$ inverse(inverse(has-sending-agent(cl,oa)) o willvolunteer(cl,i))

Assertions of the above TOVE relationships can be exploited for inference of the sources of information relevant for measuring the performance of the process elements if the following assumptions hold true: 1) Organization agent is an abstraction of an information system concept; 2) The correspondences between TOVE Information and SCOR-Full Inf-Item instances are established or inferred. 3) SCORFull Inf-Item are configured (Conf-Item) and these configurations are mapped to the corresponding goal concepts.

Alignment of SCOR-Full ontology with other relevant ontologies make all the research efforts based on these ontologies, complementary with this one, thus, improving the competence of the SCOR-Full ontology. For example, mapping of Location instances to GIS (Geographic Information Systems) ontologies can provide routing services for the shipment companies. Mapping of Product instances and corresponding identifiers to UNSPSC or eClass ontologies can enable customers to identify the suppliers of the substitutable or alternative parts or assemblies. Mapping of Process elements to Partner Interface Process instances in RosettaNet ontology can enable the collaboration between two companies using different standards for modelling and tracking their supply chain processes.

In order to improve the expressivity of the SCOR-Full ontology, it is mapped to OWL representations of TOVE ontologies (resource, organization and underlying 
activity-state-time ontologies). TOVE Resource ontology sets semantic relations (and constraints - axioms) between the notions of resource and activity. These relations enable the inference on the commitment of the resources to specific activity, their consumption and availability at given time. Thus, we can exploit the above mappings to improve the competence of SCOR-Full ontology and ask additional questions about SCOR activities, such as: Which resources are committed (or available for commitment) to a process element at given time? Is there an alternative to an unavailable resource, to be used by a process element at a given time? Or, more specific: Can the unplanned order for manufacturing of the 10 hydraulic pumps, to be delivered until September 2010, be accepted (in context of available resources)?

In addition, mappings with TOVE Organization ontology enable us to improve structural and behavioural (in context of organizational goals) competence of the SCOR-Full model. For example, answers to the following questions may become available: Whose permission (if any) is needed in order to perform the specific task of selected process element (activity)? Who has authority to verify the receipt of the sourced part? Which communication link can be used to acquire specific information? etc.

\subsection{Semantic interoperability and enterprise information systems}

The stack of the semantic technologies, consisting of informal or formal ontologies, their representations, inference engines and semantic applications, provides the means for development and implementation of a new layer of the enterprise systems architecture. The main role of this layer is to make the implicit semantics of the different existing enterprise systems (or underlying reference models) - explicit, and consequently, mutually correspondent. Thus, the layer will enable the semantic 
interoperability of these systems and facilitate better integration of the heterogeneous environments, such as Supply Chain Networks.

In this scenario, enterprise information systems will be represented in the semantic layer by local ontologies - semantically weak representations (OWL models) of the implicit knowledge related to the enterprise, and typically stored in relational databases of the relevant systems and in other data sources. Semantic matching techniques and tools can facilitate the contextualization and explicitation of the individual representations, by helping to establish the correspondences between these representations and relevant formal micro-theories, such as SCOR-Full. Consequently, semantic mappings between SCOR-Full notions and other domain and problem ontologies can be exploited for applying an integrated approach to solving some of the Supply Chain Networks issues.

For example, the partner selection problem can be associated with the definition of the individual semantic query which expresses the sufficient and necessary conditions, regarding the capability, capacity, cost, availability, etc. (SCOR-Full notion of quality) of a specific resource or an agent (among resources and agents of the whole network). Mappings between these notions, used in a query and defining correspondences between concepts in the different local ontologies, expressed as logical functions, ensure that the single query is interpreted correctly in each of the network partners' systems and corresponding data storage facilities. Thus, it becomes possible to use a single query (expressed in a formal semantics) to explore the whole supply chain network, despite the heterogeneity of used systems and their data sources (syntax, data modelling patterns, etc.).

Collaborative process management can be facilitated by monitoring the state (configurations) of the resource items in the semantic layer (by using a software 
agent), and triggering appropriate actions (e.g. initiating SCOR process elements, or equivalently, launching the process activities) when desired configurations are established. Hence, desired configurations of the resource items, whose parameters are stored as semantic annotations of the process models (generated by the process modelling tool, such as the one described in Section 3.1), are continuously compared with the specific entities of the relevant local ontologies, and logically related with those items. Once they become logically same, the software agent would assert a new individual of the Activity type, assign an agent to this individual and set other necessary properties. This change will also be propagated backwards, by assertion of the logically equivalent concepts of corresponding local ontologies and consequently, update of the relevant database(s). Thus, appropriate enterprise information system(s) will be affected by automatic insertion of the work order, web service invocation, issueing of the request for approval (authorization) or similar action.

\section{Conclusions and future work}

Although ontology matching, contextualization and other semantic web techniques provide a basis for interoperability, the challenge is still to define a whole semantic infrastructure in which supply chain participants' search for information is both relevant and efficient. In response to this challenge, this approach proposes the use of sets of semantically aligned models, on different levels of expressivity, consisting of knowledge organization system (SCOR-KOS), helper contextual models (SCOR-Cfg) and domain ontology (SCOR-Full) and, thus, potentially improving the relevance of ontology matching and facilitating contextualization.

The proposed approach relies on and builds upon widely accepted industry practice, represented in its native format (SCOR-KOS). The native representation is expected to attract attention and gain understanding of SCM experts' community and, 
hence, to facilitate the transition towards using more sophisticated, knowledge-based tools in the domain. Its mapping and alignment with higher-level ontologies (such as SCOR-Full, TOVE, and others) make the implicit, human-understandable knowledge of the supply chain operations correspondent with the explicit, machine-based representations of the relevant models and thus, corresponding systems. It enables or will enable a structured support in other relevant processes, such as resource management, partners' selection, performance tracking, exceptions handling, etc. Also, it is expected to contribute to further development and/or refinement of the SCOR reference model.

In the development of a micro-theory for supply chain operations, a bottom-up approach is applied, where existing, implicit knowledge is semantically analyzed in order to identify the specific concepts. Ontological decisions are made in the categorization of these concepts, when common general properties are recognized and used to aggregate the concepts into general notions, such as agent, course, setting, quality, function and resource items. This type of approach significantly reduces the development time, as it builds upon the already existing consensus of the domain experts, transposed into the SCOR reference model. Thus, the problem of validation of the resulting micro-theory (SCOR-Full) can be actually reduced to checking the correctness of the logical relations made between this theory's concepts and axioms and the implicit SCOR's knowledge representation (SCOR-KOS).

The main potential benefit of the SCOR-Full and associated models, including SCOR-KOS, application ontologies and the set of mappings with relevant enterprise ontologies is the facilitation of the semantic interoperability of systems in the Supply Chain Networks, in a way described in Section 4 of this paper. Multiple levels of abstraction and modular approach to the formalization of different relevant sub- 
domains and problems, applied in the development of the models are expected to improve the relevance of semantic matching results. However, there are still no evidences for this assumption. In ongoing and future efforts, we are committed to introducing the individual realities of the actual enterprises, namely, corresponding enterprise information systems, into the existing formal framework. The objective then is to show that this framework represents common reality, in context of enterprises' participation in a joint endeavour, such as the Supply Chain Network. These efforts include, but are not restricted to: generating local ontologies from the relational databases of the enterprise systems; their semantic enactment; developing methods and measures for evaluation/assessment of the semantic interoperability and development of a test bed for scenarios, briefly described in Section 4.2.

\section{References}

[1] Stewart, G., 1997. Supply-chain operations reference model (SCOR): the first cross-industry framework for integrated supply-chain management. Logistics Information Management, 10 (2), 62-67.

[2] Desai, N., Mallya, A.U., Chopra, A. and Singh, M., 2006. OWL-P: A Methodology for Business Process Development, Lecture Notes in Computer Science, 3529 79-94.

[3] Jones, A., Ivezic, N. and Gruninger, M., 2001. Toward self-integrating software applications for supply chain management. Information Systems Frontiers, 3 (4) 403-412.

[4] Lin, H.K. and Harding, J.A., 2007. A manufacturing system engineering ontology model on the semantic web for inter-enterprise collaboration. Computers in Industry, 58 (5) 428-437.

[5] Fayez, M., Rabelo, L. and Mollaghasemi, M., 2005. Ontologies for supply chain simulation modelling. In: Proceedings of the 37th Winter Simulation Conference. (Orlando, USA, December 04-07, 2005).

[6] Pathak, S.D., Nordstrom, G. and Kurokawa, S., 2000. Modelling of supply chain: a multi-agent approach. In: Proceedings of the IEEE International Conference on Systems, Man, and Cybernetics. 32051 -2056.

[7] Izza, S., 2009. Integration of industrial information systems: from syntactic to semantic integration approaches. Enterprise Information Systems, 3 (1) 1-57

[8] Ye, Y., Yang, D., Jiang, Z. and Tong, L., 2008. Ontology-based semantic models for supply chain management. International Journal of Advanced Manufacturing Technology, 37 (1112) 1250-1260

[9] Hepp, M., 2007. Possible ontologies: how reality constraints the development of relevant ontologies. IEEE Internet Computing, 11 (7) 90-96. 
[10] Guo, J., 2009. Collaborative conceptualisation: towards a conceptual foundation of interoperable electronic product catalogue system design. Enterprise Information Systems, 3 (1) 59-94.

[11] Zdravković, M. and Trajanović, M., 2009. Integrated Product Ontologies for Inter-Organizational Networks. Computer Science and Information Systems, 6 (2) 29-46.

[12] Sage, A. P., and Cuppan, D. C., 2001. On the Systems Engineering and Management of Systems-of-Systems and Federations of Systems. Information, Knowledge, Systems Management, 2 (4) 325-345.

[12] Grubic, T., Fan, I.-S., 2010. Supply chain ontology: Review, analysis and synthesis. Computers in Industry. 61 (8) 776-786.

[13] Yan, T., 2007. Practical Issues in Ontology Engineering. In: Arabnia, H.R., Yang, M.Q. Yang, J.Y, eds. Proceedings of the 2007 International Conference on Artificial Intelligence, ICAI 2007, Volume II, June 25-28, 2007, Las Vegas, Nevada, USA. pages 730-736, CSREA Press, 2007.

[14] Hepp, M., 2008. Ontologies: State of the art, business potential and grand challenges, In: Hepp, M., De Leenheer, P., De Moor, A. and Sure, Y. eds. Ontology Management - Semantic Web, Semantic Web Services and Business Applications. Berlin/Heidelberg: Springer Verlag.

[15] Guarino, N., and Giaretta,P., 1995. Ontologies and knowledge bases - towards a terminological clarification, In: Mars, N., ed. Towards very large knowledge bases: knowledge building and knowledge sharing. Amsterdam: IOS Press, 25-32.

[16] Smith, B., 2003. Ontology and Information Systems, In: Zalta, E.N., ed. The Stanford Encyclopaedia of Philosophy. Stanford, CA

[17] Noy N.F., 2004. Semantic integration: a survey of ontology-based approaches. ACM SIGMOD Record Special section on semantic integration, 33(4) 65-70

[18] Grenon, P. and Smith, B., 2004. SNAP and SPAN: Towards Dynamic Spatial Ontology. Spatial Cognition and Computation, 4 (1) 69-103.

[19] Fox, M.S., Barbuceanu, M. and Gruninger, M., 1996. An organization ontology for enterprise modelling: preliminary concepts for linking structure and behaviour. Computers in Industry, 29 (1-2) 123-134.

[20] Uschold, M., King, M., Moralee, S. and Zorgios, Y., 1998. The enterprise ontology. Knowledge Engineering Review, 13 (1) 31-89.

[21] Madni, A.M., Lin, W. and Madni, C.C., 2001. IDEONTM: An Extensible Ontology for Designing, Integrating and Managing Collaborative Distributed Enterprises. Systems Engineering, 4 (1) 35-48.

[22] Productivityappex website. http://www.productivityapex.com/products/scor.asp [Accessed 11 January 2011]

[23] Lin, Y., 2008. Semantic Annotation for Process Models. PhD thesis, Norwegian University of Science and Technology

[24] Vegetti, M., Gonnet, S., Henning, G. and Leone, H., 2005. Towards a supply chain ontology of information logistics within process industry environments. In: Proceedings of The Fourth Mercosur Congress on Process Systems Engineering, August, 14-18, 2005, Rio de Janeiro, Brazil

[25] Lu, Y., Panetto, H. and Gu, X., 2010. Ontology Approach for the Interoperability of Networked Enterprises in Supply Chain Environment. On the Move to Meaningful Internet Systems: OTM 2010 Workshops (Meersman R., et al.) (Eds)), 5th IFAC/IFIP Workshop on Enterprise Integration, 
Interoperability and Networking (EI2N'2010), Hernissou, Crete, Greece, October 27-28, Springer Verlag, Lecture Notes in Computer Science, LNCS 6428, 229-238, ISBN: 978-3-540-88874-1.

[26] Tursi, A., Panetto, H., Morel, G. and Dassisti M., 2009. Ontological approach for Products-Centric Information System Interoperability in Networked Manufacturing Enterprises. IFAC Annual Reviews in Control, 33 (2) 238-245.

[27] Millet, P.A., Schmitt, P. and Botta-Genoulaz, V., 2009. The SCOR model for the alignment of business processes and information systems. Enterprise Information Systems, 3 (4) 393-407

[28] Haller, A., Gontarczyk, J. and Kotinurmi, P., 2007. Towards a complete SCM Ontology - The Case of ontologising RosettaNet. In: Proceedings of the 2008 ACM Symposium on Applied Computing (SAC08), March 16-20, 2007, Fortaleza, Ceara, Brazil

[29] Hepp, M., 2006. Products and Services Ontologies: A Methodology for Deriving OWL Ontologies from Industrial Categorization Standards. International Journal on Semantic Web and Information Systems, 2 (1) 72-99.

[30] Hepp, M., 2006. The True Complexity of Product Representation in the Semantic Web, In: Proceedings of 14th European Conference on Information System (ECIS 2006), June 12-14, 2006, Gothenburg, Sweden

[31] Aničić, N., Ivezić, N. and Jones, A., 2005. An architecture for semantic enterprise application integration standards. In: Proceedings of The First International Conference on the Interoperability of Enterprise Software and Applications (I-ESA'05)

[32] Foxvog, D. and Bussler, C., 2006. Ontologizing EDI semantics. In: Proceedings of the Workshop on Ontologising Industrial Standards, pp. 301311. Springer, Tucson, AZ, USA, 2006

[33] Holsapple, C.W. and Joshi, K.D., 2002. A collaborative approach to ontology design. Communications of the ACM, 45 (2) 42-47

[34] Falkenhainer, B. and Forbus, K.D., 1991. Compositional modeling: finding the right model for the job. Artificial Intelligence, 51 (1-3) 95-143.

[35] Gangemi, A., 2005. Ontology Design Patterns for Semantic Web Content. Lecture Notes in Computer Science. 3729 262-276.

[36] Guarino, N., 1997. Understanding, building and using ontologies. International Journal of Human-Computer Interaction, 46 (2-3) 293-310.

[37] OWL 2 Web Ontology Language. Document Overview. W3C Recommendation 27 October 2009. http://www.w3.org/TR/owl2-overview/

[38] Zdravković, M., Panetto, H. and Trajanović, M., 2010. Concept of semantic information pool for manufacturing supply networks. International Journal of Total Quality Management and Excellence, 37 (3) 69-74.

[39] Maier, M.W., 1998. Architecting principles for systems-of-system. Systems Engineering, 1 (4) 267-284.

[40] IEEE (Institute of Electrical and Electronics Engineers), 1990. Standard Computer Dictionary - A Compilation of IEEE Standard Computer Glossaries. New York: Inst of Elect \& Electronic

[41] Panetto, H., 2007. Towards a Classification Framework for Interoperability of Enterprise Applications. International Journal of CIM, 20 (8) 727-740.

[42] Obrst, L., 2003. Ontologies for semantically interoperable systems. In: Proceedings of the 12th International Conference on Information and Knowledge Management. (New Orleans, USA, November 03 - 08, 2003) 
[43] Vernadat, F.B., 2007. Interoperable enterprise systems: Principles, concepts, and methods. Annual Reviews in Control, 31 (1) 137-145.

[44] Obrst, L., Liu, H. and Way, R., 2003. Ontologies for Corporate Web Applications. AI Magazine, Fall, 2003.

[45] Panetto H. and Molina A., 2008. Enterprise Integration and Interoperability in Manufacturing Systems: trends and issues. In: H. Panetto and A. Molina, eds. Special issue on Enterprise Integration and Interoperability in Manufacturing Systems, Computers in Industry, 59 (7) 641-646.

[46] Panetto H. (Editor), 2010. Special issue on Integration and Information in Networked Enterprises. Computers in Industry, 61 (2)

[47] Gangemi, A., Guarino, N., Masolo, C., Oltramari, A. and Schneider, L., 2002. Sweetening Ontologies with DOLCE. Knowledge Engineering and Knowledge Management: Ontologies and the Semantic Web, Lecture Notes in Computer Science, 2473 223-233. 